\title{
Essais
}

ESSAIS

Revue interdisciplinaire d'Humanités

$7 \mid 2015$

Normes communiquées, normes communicantes

\section{Traditionalisme catholique et blogosphère militante : structure et caractéristiques d'un réseau de communication}

Charlotte Blanc

\section{(2) OpenEdition \\ Journals \\ Édition électronique \\ URL : http://journals.openedition.org/essais/6285 \\ DOI : $10.4000 /$ essais.6285 \\ ISSN : 2276-0970 \\ Éditeur \\ École doctorale Montaigne Humanités}

Édition imprimée

Date de publication : 1 décembre 2015

Pagination : 66-84

ISBN : 978-2-9544269-6-9

ISSN : $2417-4211$

Référence électronique

Charlotte Blanc, «Traditionalisme catholique et blogosphère militante : structure et caractéristiques d'un réseau de communication», Essais [En ligne], 7 | 2015, mis en ligne le 26 février 2021, consulté le 27 février 2021. URL : http://journals.openedition.org/essais/6285 ; DOI : https://doi.org/10.4000/ essais. 6285 


\section{Traditionalisme catholique et blogosphère militante : structure et caractéristiques d'un réseau de communication}

\section{Charlotte Blanc}

Il est communément admis parmi les institutions et groupes religieux en tout genre qu'une visibilité numérique est le prérequis d'une communication religieuse s'inscrivant dans des réalités actuelles. À ce titre Rosalind I. J. Hackett estimait qu' " [a]ujourd'hui on est arrivé au point où une organisation religieuse paraît manquer de crédibilité, voire d'identité, si elle n'est pas présente sur le web $»^{1}$. Brenda E. Brasher ${ }^{2}$ le notait déjà dans son ouvrage précurseur Give me that online religion en se demandant comment cette présence en ligne allait changer l'avenir du fait religieux : "The question is not whether but how and when religious traditions and religious organizations will change and be changed by involvement in the online world $"^{3}$. Depuis, le champ de recherche des Internet Studies appliqué aux objets du religieux s'est considérablement développé chez les auteurs anglophones ${ }^{4}$ et plus récemment chez les auteurs

1 Rosalind I.J. Hackett, "Religion et Internet ", Diogène, n² 211, 2005, p. 88.

2 Brenda E. Brasher, Give me that online religion, New Brunswick, Rutgers University Press,

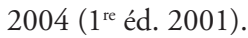

"Deeming the Internet a place that can corrode the normative way of life they promote, such leaders charge cyberspace with being anti-, rather than absent authoritarianism, a dangerous place that "true believers" either should avoid or enter only under authoritative guidance. This has led to the rise of religious intranets, religious $C D$ s, and religious search engines. Other authoritarian religious leaders worry less about what else is in cyberspace, and instead relish the Internet as a global space that can be used to convey rigidly controlled messages into the home of every online follower they can attract. Thus, the Internet has provided a new space for the exercise of religious authority ", Preface and acknowledgments, p. xiii.

3 Ibid., p. xiv.

4 Quelques références d'auteurs anglophones : Heidi Campbell, "Making space for religion in Internet Studies ", The information society, $\mathrm{n}^{\circ}$ 21(1), 2005, p. 309-315. Gad Barzilai et Karine Barzilai-Nahon, "Cultured technology: Internet and religious fundamentalism », The information society, $\mathrm{n}^{\circ} 21(1), 2005$, p. 25-40. Alf Linderman et Mia Lövheim, "Internet and Religion. The making of meaning, identity and community through computer mediated communication ", in S. Marriage and J. Mitchell (éd.), Mediating religion : conversations in media, culture and religion, Edinburgh, T\&T Clark/Continuum, 2003, p. 229-240. 
francophones ${ }^{5}$, croisant approches sociologiques en Sciences de l'information et de la communication et en Sciences sociales des religions. Internet Studies et Cultural Studies se rencontrent donc autour de l'objet religieux, réinterrogeant ce dernier dans ses dimensions organisationnelles, communicationnelles et questionnant la place du fait religieux dans l'espace public. Cette réalité concerne tant les grandes institutions religieuses que les groupes minoritaires, constituant ainsi une norme de communication en soi.

Nous cherchons à rendre compte de la présence web des traditionalistes catholiques, à travers l'observation de leurs stratégies de communication sur la blogosphère. La présence est ici entendue en tant que " présence numérique " proposée par Louise Merzeau ${ }^{6}$ comme ensemble de traces (entités informationnelles) laissées par les utilisateurs au gré des connexions, qui laissent apparaître des éléments de leur identité 7 . Le " mariage pour tous ${ }^{8}$ (2012-2013) constitue ici un outil de sélection du corpus. L'instauration d'un nouvel article dans le code civil, ouvrant l'institution du mariage aux personnes de même sexe a suscité une mobilisation importante de la part des citoyens, en particuliers des croyants. Les traditionalistes ont pris part à cette mobilisation "manif pour tous ". L'aspect polémique du projet de loi, sa visibilité (et longévité) médiatique ainsi que l'implication des groupes religieux qu'il a suscité permettent d'aborder les réseaux traditionalistes par ce prisme. De plus, les mobilisations notables des traditionalistes lors de la loi sur le PACS nous semblaient inscrire la question du " mariage pour tous » en continuité de la doxa traditionaliste concernant l'évolution des mœurs. Enfin, l'intérêt porté à un seul événement médiatique est une bonne méthode pour appréhender le terrain, plutôt que d'opter pour une exploration " flottante " de celui-ci. Le traditionalisme est entendu ici dans ses acceptions historique et sociologique. Ainsi en tant que notion sociologique le traditionalisme concerne des courants religieux qui « agissent généralement à l'intérieur du seul champ religieux, tentant de maintenir indemne l'héritage de la tradition originelle. Leur action vise directement les coreligionnaires qui auraient dénaturé l'orthodoxie religieuse de la tradition par un trop grand nombre de compromis avec le

Jeff Zaleski, The soul of cyberspace: How technology is changing our spiritual lives, San Francisco, HarperSanFrancisco, 1997.

5 Jean-François Mayer, Internetet Religion, Gollion, infolio, 2008. David Douyère, Stéphane Dufour et Odile Riondet (éd.), Religion et communication, MEI, nº 38, Paris, L'Harmattan, 2014.

6 Louise Merzeau, «Du signe à la trace : l'information sur mesure », Hermès, n ${ }^{\circ 9}$, "Traçabilité et réseaux ", 2009, p. 23-29.

7 L'identité renvoie ici à la typologie sémiotique dressée par Fanny Georges dans « Représentation de soi et identité numérique. Une approche sémiotique et quantitative de l'emprise culturelle du web 2.0 ", Réseaux, n 154, 2009/2, p. 165-193. Fanny Georges identifie trois entités : une identité déclarative, une identité agissante et une identité calculée.

8 "Mariage pour tous " est le vocable employé pour qualifier la loi du 18 mai 2013 porté par la ministre Christiane Taubira et ouvrant les droits du mariage aux personnes de même sexe. http://www.gouvernement.fr/action/le-mariage-pour-tous [consulté le 3/09/2014]. 
monde moderne $"{ }^{9}$. Le traditionalisme se compose de différentes mouvances catholiques nées d'une opposition au tournant de la modernité, amorcée sous plusieurs formes dès le début du $\mathrm{XX}^{\mathrm{e}}$ siècle $^{10}$ et prenant racine chez le très réformiste concile Vatican II. Ces différentes mouvances (intransigeantisme, lefebvrisme ${ }^{11}$, sédévacantisme, ou traditionalistes intégrés au giron de l'Église - communautés rassemblées sous la commission Ecclesia Dei, puis Sommorum Pontificum ${ }^{12}$ ) manifestent toutes leur désaccord plus ou moins fort avec les réformes progressistes impulsées par le Concile (notamment sur la liberté religieuse). En découle une reconnaissance de l'autorité du pape (et de l'infaillibilité pontificale ${ }^{13}$ ) à géométrie variable selon les courants. Ces mouvances ont un attachement fort à la Tradition catholique dont ils font une lecture sélective et anti-moderniste. Le traditionalisme renvoie à ce que Gérard Lenclud qualifie d'" absence de changement dans un contexte de changement $~^{14}$ et à un attachement particulier au " dépôt culturel sélectionné ${ }^{15}$ que représente la Tradition telle que définie ante Vatican II.

Ainsi, nous nous posons les questions suivantes. Sur quels éléments se structurent les milieux traditionalistes sur Internet? Quels en sont les dispositifs et acteurs? Nous cherchons à rendre compte de la structuration du réseau sur la base des interactions entre les sites et blogs traditionalistes. Quelles sont les caractéristiques structurelles de ces réseaux ? Quelles en sont les normes de communication, entendues comme hégémonie de points de vue, en termes de contenu ? Nous nous sommes intéressées à la structure du réseau traditionaliste par le prisme du " mariage pour tous " lors de notre recherche exploratoire

9 Cf. Micheline Milot, "Religion et intégrisme, ou les paradoxes du désenchantement du monde ", Cabiers de recherche sociologique, $\mathrm{n}^{\circ}$ 30, 1998, p. 153-178.

10 Voir Émile Poulat, Intégrisme et catholicisme intégral, un réseau secret international antimoderniste: La "Sapinière ", 1909-1921, Paris, Casterman, 1969.

11 Mouvement crée sous l'impulsion de Monseigneur Marcel Lefebvre fondateur de la Fraternité Sacerdotale Saint Pie X, érigée en novembre 1970 à l'issue de l'aggiornamento entrepris par le Concile Vatican II. La fraternité deviendra dissidente de l'Église en 1988 suite à l'ordination de quatre évêques par Mgr Lefebvre sans accord pontifical, provoquant ainsi un "schisme " dans l'Église. Voir Luc Perrin, L'affaire Lefebure, Paris/Montréal, Cerf/Fides, collection "Bref ", 1989, chapitre I "Genèse d'un schisme ", p. 11 ; Nicolas Senèze, La crise intégriste, Vingt ans après le schisme de Mgr Lefebure, Paris, Bayard, 2008, chapitre 6 "Le schisme ", partie 1 " La radicalisation ", p. 105. Voir également Yves Congar, La crise dans l'Église et Mgr Lefebure, Paris, Cerf, 1976.

12 Site web du Vatican, sur la commission Ecclesia Dei, http://www.vatican.va/roman_curia/ pontifical_commissions/ecclsdei/documents/hf_jp-ii_motu-proprio_02071988_ecclesia-dei_ en.html [consulté le 25/09/2014].

13 Voir Encyclopeadia Universalis, http://www.universalis.fr/encyclopedie/infaillibilite-pontificale/, [consulté le 15/02/2015].

14 Cf. Lenclud Gérard, "La tradition n'est plus ce qu'elle était... Sur les notions de tradition et de société traditionnelle en ethnologie ", Terrain, $\mathrm{n}^{\circ}$ 9, " Habiter la Maison ", octobre 1987, p. 110-123.

15 Idem. 
au travail de thèse. Ignorant à ce stade l'étendue du terrain traditionaliste sur le web, de même, par peur d'une infobésité ${ }^{16}$, il nous a semblé en ce temps que l'explorer par le prisme d'une série d'événements en lien avec un thème précis pouvait être éclairant sur les pratiques de communication et de structuration d'un discours et de l'action des traditionalistes catholiques. La démarche a permis de mettre en place une méthode de collecte efficace. Ainsi, dans une approche systémique, à la fois analytique et interactionniste, nous nous proposons ici de traiter de la mobilisation traditionaliste dans ses dimensions symboliques (faisant appel à un socle de références idéologiques et à un imaginaire de la Tradition ${ }^{17}$ ) et communicationnelles. Nous chercherons ainsi à observer l'existence d'une homogénéité ou d'une hétérogénéité dans les formes de discours à travers la mise en évidence des interactions entre les dispositifs du web traditionaliste et des revendications de leurs acteurs. Le développement se fera en trois temps. Nous nous intéresserons en premier lieu à la morphologie du réseau traditionaliste sur Internet, puis nous ciblerons notre travail sur la blogosphère traditionaliste, pour enfin s'intéresser en dernière partie aux caractéristiques du discours traditionaliste.

\section{Morphologie des réseaux traditionalistes}

\section{Méthodologie de recherche}

Nous optons pour une démarche d'observation ethnographique sur le terrain internet afin de mettre en évidence la morphologie des réseaux de communication traditionalistes en nous focalisant sur les interactions entre les différentes plateformes. Notre démarche est ethnographique en ce sens qu'elle vise à faire une "étude descriptive et analytique des écrits numériques des internautes ${ }^{18}$. Pour ce faire nous nous inspirons de travaux récents sur

16 Terme qui renvoie à une surcharge informationnelle sur Internet et à l'organisation de l'information. Voir Samy Ben Amor et Lucia Granget, "L'infobésité, un phénomène au détriment de la qualité informationnelle ", Imad Saleh et al. (coord.), Pratiques et usages numériques, H2PTM'13, Paris, Hermès Sciences Publications, Lavoisier, 2013.

17 Afin d'éclairer davantage le propos sur ce concept de Tradition perçu et entendu par les catholiques se revendiquant de la Tradition, une explication de Yves Congar est pertinente : "La Tradition, pour lui (Mgr Lefebvre), est ce qu'il appelle " la Rome éternelle », « la messe de toujours », c'està-dire Rome jusqu’à Paul VI exclusivement, jusqu'au missel de 1969 exclusivement. [...] En se référant sans cesse à la messe de saint Pie V (1570), au catéchisme du concile de Trente (1566), à celui de saint Pie X (1912), Mgr Lefebvre ne fixe-t-il pas la Tradition dans des formules du passé, certainement saintes et valables mais qui, au plan de la formulation, ne peuvent interdire de chercher une meilleure adaptation aux besoins d'aujourd'hui ? ", op. cit., p. 56-57.

18 Voir Josiane Jouët et Coralie Le Caroff, "L'observation ethnographique en ligne ", Manuel d'analyse du Web, Christine Barats (éd.), Paris, Armand Colin, Collection U, Sciences humaines et sociales, 2013, p. 147. 
la participation citoyenne et politique en ligne ${ }^{19}$ et nous nous inscrivons en particulier dans la lignée des link studies, soit l'observation de la mobilisation en ligne par liens hypertextes. Ainsi notre recherche s'appuie sur une approche qui utilise le lien hypertexte comme outil d'analyse (et ressource) de la mobilisation en ligne ${ }^{20}$. Nous souhaitons de la sorte cibler les lieux de production de l'information sur le " mariage pour tous " sur les réseaux traditionalistes ainsi que les dynamiques de circulation de l'information. Nous avons employé la méthode d'échantillonnage de réseau ${ }^{21}$ pour laquelle deux indicateurs ont été sélectionnés. Il s'agit en premier lieu de l'appartenance au catholicisme traditionaliste, indicateur pour lequel une grille de lecture a été faite montrant les critères théologiques, liturgiques, idéologiques, des traditionalistes catholiques dans leur aspect " œcuménique ». Le second critère était le clair positionnement contre le «mariage pour tous ». L'objectif de ce premier point est de mettre en avant les particularités des réseaux traditionalistes sur internet par le prisme des sites catholiques anti-mariage pour tous. Notre étude offre donc une vue de ces réseaux d'opposition et de mobilisation.

La difficulté première a donc été de dresser une grille de lecture efficace et représentative, car tous les regroupements religieux forment des " groupes sociaux " caractérisés par des frontières floues: l'appartenance au groupe se définit par des critères formels rigides que dans le cas de groupes reclus d'élus, de purs, de saints. Même parmi les membres dits actifs, il existe généralement des degrés d'appartenance, de référence et d'engagement. Ceci a une conséquence bien évidente pour toute approche organisationnelle puisqu'il s'avère difficile de dénombrer les membres d'une église, d'une confession, d'un culte, d'une dénomination, voire d'une secte. C'est bien pour cette raison que les sciences sociales des phénomènes religieux ne pouvant pas parler $\mathrm{d}^{\text {' }}$ entreprises religieuses ", voire d'organisations religieuses, ont principalement centré leur attention sur le clergé ou les leaders religieux en tant que membres permanents ${ }^{22}$.

19 À ce sujet consulter l'article de Laurence Monnoyer-Smith sur l'état de l'art « La participation en ligne, révélateur d'une évolution des pratiques politiques ? ", Participations, 2011/1, excellente revue sur les rapports entre culture numérique et nouveaux espaces délibératifs et les approches, françaises principalement, de l'analyse de la participation politique en ligne. À consulter également les travaux de Laurence Allard et Olivier Blondeau, notamment " L'activisme contemporain : défection, expressivisme, expérimentation ", Rue Descartes, Collège international de philosophie, $\mathrm{n}^{\circ}$ 55, 2007/1, p. 47-58 ; ou encore Romain Badouard « Les mobilisations de clavier. Le lien hypertexte comme ressource des actions collectives en ligne ", Réseaux, n 181, 2013/5, Paris, La Découverte, p. 87-117, sur l'aspect méthodologique de l'analyse de la mobilisation en ligne, réinterrogeant ainsi les théories classiques de l'action collective.

20 Idem, Voir Romain Badouard.

21 Échantillonnage de réseaux : échantillonnage empirique, les sites sont sélectionnés en fonction des liens qu'ils entretiennent avec un site-noyau.

22 Paul Stryckman, "La communication des institutions religieuses ", Communication et organisation, $\mathrm{n}^{\circ}$ 9, 1996, p. 2-3. 
Quels sont les critères " formels " dans le cas qui nous intéresse ? Le corpus de sites étudiés s'est construit à partir d'une grille de lecture du traditionalisme établie sur l'état de l'art concernant le traditionalisme et l'intégrisme catholiques ${ }^{23}$.

Le corpus a été établi en partant des sites Internet des institutions cultuelles, telles que la Fraternité Sacerdotale Saint Pie X, l'Institut du Christ Roi ou la Fraternité Sacerdotale Saint Pierre. Ces sites ont été le point de départ de notre recherche, et choisis en raison de leur appartenance formelle et certaine au traditionalisme catholique. Ainsi, nous souhaitions avoir une approche institutionnelle du traditionalisme.

Toujours selon Stryckman «le groupe religieux évolue selon un développement organisationnel de type "normatif $»^{24}$. Or, sommes-nous ici en présence d'un groupe religieux structuré autour de normes diffusées par une institution sur le web ? C'est ce que nous avons essayé de savoir à travers une observation sur la base des blogrolls des sites web et blogs des institutions. En effet, la démarche de s'intéresser aux blogrolls permet de comprendre, entre autres, dans quel champ de références gravitent les sites traditionalistes, de voir quelles sont leurs recommandations en matière de blogs. Ainsi, toutes ces données ont été recoupées et nous ont menées vers un ensemble de blogs largement consultés. Il s'agissait ensuite de vérifier l'appartenance confessionnelle et idéologique de ce corpus de blogs à partir de la grille de lecture. De même, les sites ou blogs ne correspondant pas aux critères préétablis n’ont pas été sélectionnés pour le corpus.

Toutefois, une observation par blogrolls est insuffisante à la mise en évidence d'un réseau de sites interconnectés ; c'est pourquoi une collecte des liens hypertextes a également été menée. Chaque site ou blog trouvé par le biais de l'observation par blogrolls a été entré dans un crawlerr $^{25}$. La méthode permet un regroupement de sites web par le biais de liens hypertextes; le crawler est " un robot qui explore et indexe les sites web en laissant le chercheur guider la navigation $»^{26}$. Enfin, l'ensemble des sites a pu ensuite être visualisé grâce au logiciel Gephi ${ }^{27}$ (le logiciel Gephi est utilisé pour la visualisation de réseaux). Le corpus conservé pour l'étude de cas présente s'est limité à 60 sites traditionalistes ou d'affinité traditionaliste.

23 Luc Perrin, idem; Nicolas Senèze, idem; Yves Congar ; idem; Émile Poulat, idem; Henri Tincq, Catholicisme, Le retour des intégristes, Paris, CNRS Éditions, 2009; René Rémond, «L'intégrisme catholique. Portrait intellectuel ", Études, 370, n 1, Paris, 1989, p. 95-105; Florian Michel et Bernard Sesboüé, De Mgr Lefebvre à Mgr Williamson, Anatomie d'un schisme, Paris, Lethielleux, DDB, 2009.

24 Op. cit., p. 3.

25 Sur la méthode de collecte par liens hypertextes voir Méthodologies d'analyse de corpus en Sciences humaines à l'aide du Navicrawler, Mathieu Jacomy, Franck Guitalla, Rapport final 2007. Crawler utilisé ici : Plugin Firefox Navicrawler.

26 Voir Jean-Christophe Plantin, « D’une carte à l'autre : Le potentiel heuristique de la comparaison entre graphe du web et carte géographique ", in Christine Barats (éd.), op. cit., p. 229.

27 http://gephi.github.io/ [consulté le 29/03/2015]. 


\begin{tabular}{|c|c|c|}
\hline DIMENSIONS & INDICATEURS & VARIABLES \\
\hline \multirow[t]{2}{*}{ Théologique } & Théologie du Christ-Roi & \\
\hline & Théologie morale de Léon XIII & \\
\hline \multirow[t]{2}{*}{ Ritualiste } & Messe tridentine & \\
\hline & Service de l'autel (dos aux fidèles) & \\
\hline \multirow[t]{2}{*}{ Catéchistique } & Références au concile de Trente & \\
\hline & Références aux papes ante Vatican II & Principalement : Pie X, Léon XIII \\
\hline \multirow[t]{5}{*}{ Apologétique } & Défense de l'Église et de la foi & $\begin{array}{c}\text { Débat autour de la question } \\
\text { de l'« infaillibilité pontificale » }\end{array}$ \\
\hline & «Combat » de la Tradition & Contre Vatican II de façon générale \\
\hline & & Contre l'œcuménisme \\
\hline & & Contre la liberté religieuse \\
\hline & & Évocation d'une crise dans l'Église \\
\hline \multirow[t]{3}{*}{$\begin{array}{l}\text { Utopiste, idéal } \\
\text { catholique }\end{array}$} & «Cité catholique» & Doctrine sociale et morale chrétienne \\
\hline & Rejoint les dimensions politiques & \\
\hline & $\begin{array}{l}\text { Rejoint les dimensions } \\
\text { apologétiques }\end{array}$ & \\
\hline \multirow[t]{2}{*}{ Martyrologique } & $\begin{array}{l}\text { Expression d'un sentiment de } \\
\text { persécution ou d'injustice }\end{array}$ & Personnages incarnant le martyr \\
\hline & $\begin{array}{l}\text { Références à des « martyrs " } \\
\text { catholiques }\end{array}$ & \\
\hline \multirow[t]{5}{*}{ Conspirationniste } & Franc-maçonnerie & \\
\hline & Judaïsme & \\
\hline & Communisme & \\
\hline & $\begin{array}{l}\text { Oppositions autres n'appartenant } \\
\text { pas à une quelconque " théorie " } \\
\text { du complot }\end{array}$ & Charismatisme, Opus Dei... \\
\hline & & $\begin{array}{l}\text { "Fausses religions » : Protestantisme, } \\
\text { Islam, Orthodoxie }\end{array}$ \\
\hline \multirow[t]{2}{*}{ Symbolique } & Emblèmes catholiques de Tradition & Cœur de Jésus, Christ-Roi, Glaive... \\
\hline & Lieux de pèlerinages & Ex. Chartres pour la Pentecôte \\
\hline \multirow[t]{3}{*}{ Politique } & Nationalisme & \\
\hline & Royalisme & Ex. Action française, Manants du roi... \\
\hline & Légitimisme & \\
\hline \multirow[t]{2}{*}{$\begin{array}{c}\text { Journalistique/ } \\
\text { Communicationnelle }\end{array}$} & Confessionnel & $\begin{array}{l}\text { Ex. Itinéraires, Minute... } \\
\text { Bulletins de paroisse }\end{array}$ \\
\hline & Professionnel/Politique & Ex. Rivarol, Présent, Action française... \\
\hline
\end{tabular}

Tableau 1 : Grille de lecture synthétisée du traditionalisme catholique

\section{Typologie du web traditionaliste}

Une typologie des sites et blogs du corpus a été dressée afin de saisir le paysage traditionaliste, les dispositifs et les acteurs du réseau. Ci-dessous 
nous présentons un extrait de trois sites, Renaissance Catholique, Civitas et Riposte Catholique. Les différentes variables de l'analyse des sites sont le dispositif employé, la visée info-communicationnelle, les objectifs clairement annoncés du site, le(s) thème(s) qui ressort(ent) le plus lors d'une première lecture du site, l'orientation religieuse (qui confirme l'appartenance du site au traditionalisme catholique). L'avant-dernière rubrique permet d'inscrire le site dans un réseau d'acteurs d'une part, en donnant le nom des sites et blogs listés dans le blogroll, d'autre part elle précise les acteurs impliqués dans la gestion du site ou blog (laïcs, association, institution, etc.).

\begin{tabular}{|c|c|c|l|}
\hline & DISPOSITIF & VISÉE & \multicolumn{1}{|c|}{ OBJECTIFS PREMIERS } \\
\hline $\begin{array}{c}\text { Renaissance } \\
\text { catholique } \\
(2010)\end{array}$ & $\begin{array}{c}\text { Site natif } \\
\text { de l'Internet }\end{array}$ & $\begin{array}{c}\text { Informationnelle } \\
\text { Collaborative }\end{array}$ & $\begin{array}{l}\text { Information sur les enseignements et la } \\
\text { liturgie traditionnels de l'Église catholique/ } \\
\text { Actualité catholique et politique. }\end{array}$ \\
\hline $\begin{array}{c}\text { Civitas } \\
(2009) \\
\text { Alain Escada }\end{array}$ & $\begin{array}{c}\text { Site natif } \\
\text { de l'Internet }\end{array}$ & $\begin{array}{c}\text { Informationnelle } \\
\text { Collaborative }\end{array}$ & $\begin{array}{l}\text { Informer et former sur la doctrine sociale de } \\
\text { l'Église ; rechristianisation de la France. } \\
\text { Communiquer pour convaincre. }\end{array}$ \\
\hline $\begin{array}{c}\text { Riposte } \\
\text { catholique } \\
(2012)\end{array}$ & Blog portail & $\begin{array}{c}\text { Informationnelle } \\
\text { Communicationnelle }\end{array}$ & $\begin{array}{l}\text { Portail d'information visant à rassembler les } \\
\text { actualités catholiques en un même endroit. }\end{array}$ \\
\hline
\end{tabular}

\begin{tabular}{|c|c|c|}
\hline $\begin{array}{c}\text { THÈMES FAVORIS } \\
\text { (en lecture flottante } \\
\text { et au moment de l'étude) }\end{array}$ & $\begin{array}{l}\text { ORGANISATION ET LIENS } \\
\text { SUR LES RÉSEAUX SOCIAUX }\end{array}$ & $\begin{array}{l}\text { ORIENTATION } \\
\text { RELIGIEUSE }\end{array}$ \\
\hline $\begin{array}{l}\text { Mariage pour tous } \\
\text { Manifestations } \\
\text { anti-chrétiennes }\end{array}$ & $\begin{array}{l}\text { Association } \\
\text { Revue }\left(5 \mathrm{n}^{\circ} / \mathrm{an}\right) \\
\text { Laïcs } \\
\text { Réseau Renaissance : Cercle renaissance }\end{array}$ & Catholiques de tradition \\
\hline $\begin{array}{l}\text { Manifestations } \\
\text { anti-chrétiennes } \\
\text { Mariage pour tous }\end{array}$ & $\begin{array}{l}\text { Institut de formation } \\
\text { Revue d'actualité politique et religieuse } \\
\text { Laïcs } \\
\text { Réseau Civitas, France jeunesse civitas } \\
\text { Twitter Facebook }\end{array}$ & Catholiques de tradition \\
\hline $\begin{array}{l}\text { Catholicisme de tradition } \\
\text { Mariage pour tous } \\
\text { Manifestations } \\
\text { anti-chrétiennes }\end{array}$ & $\begin{array}{l}\text { Réseau d'information: } \\
\text { - Osservatore Vaticano } \\
\text { - Jeanne Smits (Présent) } \\
\text { - Observatoire de la christianophobie } \\
\text { - Itinerarium } \\
\text { - Laïcs } \\
\text { Twitter, Facebook, Youtube, RSS }\end{array}$ & Catholiques de tradition \\
\hline
\end{tabular}

Tableau 2 : Typologie des sites et blogs du corpus (extrait)

Cette première lecture des sites et blogs traditionalistes a été faite pour l'ensemble du corpus. L'étape a permis de vérifier l'appartenance, de prendre connaissance des acteurs et des liens que ces acteurs entretiennent entre eux et de les inscrire dans un réseau d'information. De ces précédentes démarches nous avons pu extraire une première morphologie du web traditionaliste que nous allons exposer. 


\section{« Réinfosphère » et spécificités du réseau traditionaliste}

\section{Hétérogénéité du web traditionaliste}

À la suite de la typologie dressée, nous avons pu mettre en évidence les types d'acteurs impliqués dans la production blogging chez les traditionalistes et rendre compte d'une certaine hétérogénéité. Les acteurs en question appartiennent principalement au domaine associatif (hors institutions religieuses) et à la presse semi-professionnelle voire amateur. Nous pouvons dès maintenant constater une segmentation au sein du réseau traditionaliste en plusieurs domaines d'intérêts et subdivision de réseaux. La blogosphère comporte premièrement une sphère spirituelle et d'entraide sous l'autorité des institutions cultuelles. Les usages de cette sphère sont multiples et vont de la diffusion de matière théologique et spirituelle à de l'entraide entre traditionalistes (Entraide catholique $e^{28}$ par exemple). Se trouvent également dans cette sphère des espaces d'échange (Forum catholique ${ }^{29}$ ) ou des sites de petites annonces destinées aux ménages traditionalistes. Ainsi cette sphère a plusieurs fonctionnalités, dont celle d'accompagner les fidèles, du quotidien au lieu de culte, en passant par l'enseignement religieux. Elle permet également aux fidèles de se repérer dans l'espace traditionaliste français à travers le réseau MISSA $^{30}$ qui recense tous les lieux de culte de la mouvance traditionaliste, quelle que soit la tendance.

La blogosphère traditionaliste se compose également d'une sphère politique dans laquelle plusieurs affinités politiques se dessinent : des royalistes, des maurrassiens avec l'Action française ${ }^{31}$ par exemple et des identitaires (Novopress ${ }^{32}$ ). Enfin, dans la mouvance de Rivarol émerge une tendance plus historique, négationniste et antisémite. Cette sphère politique n'est pas sans ressemblance avec la typologie dressée par le journaliste David Doucet ${ }^{33}$ sur la blogosphère française d'extrême droite. Une dernière sphère a retenu notre attention, c'est la blogosphère militante associative qui se compose de nombreux collectifs associatifs et dont l'objet est la défense d'une cause (concernant principalement les thèmes suivants : l'euthanasie, les mouvements pro-vie, l'enfance, le mariage entre personnes de même sexe). Au centre de ces trois pôles d'appartenance au traditionalisme catholique se trouve une dernière sphère qui nous intéresse tout particulièrement, l'auto-désignée " réinfosphère ", composée d'une vingtaine de blogs.

28 http://www.entraide-catholique.org/site/index.php [consulté le 01/09/2014].

29 http://www.leforumcatholique.org/ [consulté le 01/09/2014].

30 http://www.missa.fr/. Le réseau MISSA est présenté par le site Entraide Catholique comme un partenaire de la Fraternité Sacerdotale Saint Pie X et Civitas comme un "site ami » [consulté le $01 / 09 / 2014]$.

31 http://www.actionfrancaise.net/craf/ [consulté le 01/09/2014].

32 http://fr.novopress.info/ [consulté le 01/09/2014].

33 http://www.dailymotion.com/video/xr4vin_france-culture-2012-05-26-blogosphere-extreme-droite_news. David Doucet, journaliste aux Inrocks [consulté le 01/09/2014]. 


\section{La « réinfosphère » traditionaliste : un organe de réinformation}

L'emploi du terme « réinfosphère " est passé dans le langage couramment usité sur la blogosphère traditionaliste. Une analyse de contenu sera, lors de prochaines recherches, la bienvenue. Nous avons pu mettre en avant l'hétérogénéité qui caractérise le réseau traditionaliste. De cette hétérogénéité émerge néanmoins un pôle informationnel central, la réinfosphère. L'analyse des liens hypertextes et les listes de blogrolls dressées pour chacun des sites du corpus a mis en évidence une vingtaine de blogs " conseillés " par les autres sites traditionalistes. Ces derniers sont directement alimentés par cette "réinfosphère " qui constitue une blogosphère à visée réinformationnelle. Cet objectif de " réinformation " est clairement mentionné sur de nombreux sites, notamment à travers les intitulés choisis ("Contre-Info »- Renouveau français -, « Riposte Catholique : portail de réinformation au quotidien " par exemple). Le choix du terme est stratégique et témoigne de l'intentionnalité des acteurs traditionalistes laïcs de fournir une autre information, une information à visée alternative. Ce qui n'apparaissait pas évident au début de la collecte est devenu plus clair : il n'est pas certain que les acteurs se soient initialement concertés pour la création de ces blogs d'information. En revanche, l'accumulation de mentions de tel ou tel blog dans le blogroll d'un site "ami " est significatif : quantitativement d'une part, puisqu'il témoigne de l'importance d'un blog en tant que "référence " dans le réseau ; qualitativement ensuite, puisque cela signifie que le blog en question fournit de l'information, appréciée par l'ensemble du réseau.

L'intention communicationnelle est clairement exprimée. Ainsi, nous prenons pour exemple la présentation du site Novopress: Novopress se donne pour objectif de refaire l'information face à "l'idéologie unique ". Il en va de même pour le site de la Contre-Info dont l'objectif est d'aller à l'encontre de l'information "dominante "; ou encore le site TradiNews, qui marque par son titre sa volonté de fournir un traitement médiatique de l'information adapté et à destination d'une population traditionaliste. La présentation de Novopress suggère même un travail à caractère coopératif parmi les acteurs de l'information traditionaliste: «Mais ce travail de réinformation ne peut pas se faire seul. La complémentarité entre les différentes plateformes doit permettre de développer un véritable écosystème réinformationnel ॥ $^{34}$. L'expression même d' « écosystème réinformationnel » exprime explicitement la volonté de fournir un média alternatif, voire un "système " médiatique alternatif puisqu'il prône la complémentarité. Les rubriques proposées sur les blogs sont représentatives des intentions de leurs concepteurs. Le site contre-info par exemple propose des rubriques telles que : "Résistance ", "Lobbies et secte » ou "Bourrage de

34 Les « différentes plateformes » auxquelles Novopress fait allusion sont : FdeSouche, Polémia, Nouvelles de France, Info Bordeaux et Minute. 
crânes/manipulations " indiquant un sentiment de manipulation de la part des médias dominants. Des sites tels que celui-ci ont donc vocation de fournir une information alternative à celle diffusée par les médias classiques, assimilés souvent à une orchestration maçonnique. L'objectif du site est de donner " accès à une information libre, loin des manipulations, diversions et bobards médiatiques ${ }^{35}$. Ainsi, nous nous sommes demandées si les dispositifs sociotechniques du réseau traditionaliste produisent de l'information militante. Dans une logique médiactiviste, cette réinfosphère correspond rait à une critique contre-hégémonique. Le terme hégémonique s'inscrit en remplacement du terme d'idéologie propre aux travaux de L'idéologie allemande (Marx, Engels) et de La dialectique de la raison (Horkheimer et Adorno), et exprime le passage d'un paradigme de la domination dans les rapports sociaux à celui de pouvoir. L'hégémonie (et contre-hégémonie) est l'objet de recherche issu du tournant gramscien et de l'apport post-critique des culturalstudies. Le terme, emprunté à Antonio Gramsci par Stuart Hall et Raymond Williams ("Cultural studies and its theoretical legitimacies ") renvoie à l'existence d'un point de vue majoritaire et de points de vue minoritaires, en perpétuel conflit de représentation dans la sphère publique. Le nouvel objet de ce tournant postcritique est "l'analyse des mouvements et des contre-mouvements culturels dans la sphère publique ${ }^{36}$. La critique contre-hégémonique renvoie donc selon John Downing, à " une vision alternative aux programmes politiques, aux priorités et aux perspectives hégémoniques ${ }^{37}$ constituant la caractéristique propre des médias radicaux. Quant au médiactivisme suggéré ici, il est l'expression médiatique de conflits culturels, d'exposition d'un point de vue minoritaire face à une hégémonie culturelle manifeste dans les médias de masse, par l'usage alternatif des médias ou de médias alternatifs.

C'est parce que la sphère publique n'est pas égalitaire que certains groupes sociaux ou certains points de vue doivent d'abord se constituer en " contrepublics subalternes " et développer au sein d'un réseau propre (ayant souvent leurs propres supports médiatiques) la définition de leurs adversaires, de leur identité et des " problèmes » devant être pris en compte au niveau plus général ${ }^{38}$.

La blogosphère est un réseau d'influence fournissant une " matière idéologique " au corps associatif, elle prend un caractère contre-hégémonique en ce sens qu'elle est un organe d'information et un outil de mobilisation. Elle produit une mobilisation informationnelle destinée à mettre en évidence les

35 http://www.contre-info.com/category/contre-info [consulté le 22/01/2015].

36 Éric Macé, Les imaginaires médiatiques, Une Sociologie postcritique des médias, Paris, Éditions Amsterdam, 2006, p. 16.

37 John D. H. Downing, Radical Media. Rebellious communication and social movements, Londres, Sage, 2001, p. 16.

38 Éric Macé, op. cit., p. 90. 
influences subies par les médias dominants ${ }^{39}$. À travers le thème du "mariage pour tous " nous cherchons à observer les dispositifs blogs impliqués discursivement dans une forme de mobilisation. Le " mariage pour tous " n'est qu' un axe possible de recherche sur les mobilisations traditionalistes, l'objectif à plus grande échelle sera d'observer la structuration de la mobilisation sous toutes ses formes et dans la variété des causes et sujets défendus. Il est donc, encore une fois, un point d'entrée dans le réseau d'information et de mobilisation, pour saisir dans un premier temps les caractéristiques normatives du discours. Nous aurions pu entreprendre une analyse micro-logique et nous intéresser à un site en particulier, ou un groupe spécifique, or nous avons choisi de procéder à une analyse plus large, afin de saisir une dynamique d'ensemble ${ }^{40}$.

La mobilisation informationnelle que génèrent les interactions sur les réseaux est animée par une logique qui se structure sur la base d'un modèle idéal de vie partagé et un positionnement politique à l'extrême droite de façon générale. L'intérêt porté au dispositif "blog " prend ici tout son sens. Il est en effet producteur d'une identité narrative prolifique, il laisse au producteur une marge importante d'expression d'une identité déclarative (ce que je dis et ce que je donne de moi), ce qui permet au chercheur de prendre la mesure des intentions et représentations de l'internaute. L'un des enjeux de cette recherche est de saisir le système de représentations de cette blogosphère de " réinformation ", cadre d'énonciation de la Tradition catholique. Nous tentons de comprendre ici ce qui est à l'origine des liens unissant les traditionalistes dans la défense d'un modèle familial. Leur système de représentations constitue-t-il une force mobilisatrice, un réservoir symbolique générant de l'action ? Nous pouvons ajouter que " pour l'utilisateur, l'attrait des blogs, des plateformes de partage et des réseaux sociaux consiste dans la mise en commun de ses marqueurs individuels (centre d'intérêt, préférences, commentaires, statuts, etc.) $»^{41}$. Ce sont des " marqueurs individuels» qui s'inscrivent dans une traçabilité de l'individu et des éléments de son identité, phénomène observable notamment par l'étude du lien hypertexte.

39 Voir Dominique Cardon et Fabien Granjon, Médiactivistes, Paris, Presses de Science Po, 2013

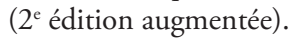

40 Anne Bellon explique l'intérêt de l'approche dans son article "S'engager sur Internet. La mobilisation en ligne de la communauté informatique ", Politiques de communication, $\mathrm{n}^{\circ} 3$, "S'engager sur Internet. Mobilisations numériques et pratiques politiques », automne 2014, Grenoble, PUG.

41 Louise Merzeau, «Présence numérique : les médiations de l'identité ", Les enjeux de l'information et de la communication, 2009, p. 79-91. 


\section{Une mobilisation ré-informationnelle}

\section{Une mobilisation symbolique}

L'analyse de contenu s'est effectuée sur la base de documents textuels et visuels de la blogosphère. Elle est centrée sur la production manifeste du discours $^{42}$. De ce fait nous avons sélectionné textes et vidéos porteurs d'une opposition au « mariage pour tous » datant de la période d'ouverture du débat, à la promulgation de la loi (7 novembre 2012-18 mai 2013). Les textes et vidéos ont été traités par la méthode d'analyse logico-sémantique thématique suivant les principes de l'analyse automatique. L'ensemble des discours se structure sur des représentations dualistes et un découpage entre les éléments appartenant à l'univers du Bien et ceux de celui du Mal. Ci-dessous nous proposons un extrait des grands thèmes recensés sur le " mariage pour tous " à travers un tableau schématique.

\begin{tabular}{|c|c|}
\hline BIEN « Nous » Victimes & MAL « Eux » Responsables \\
\hline Juste, vrai, vérité & Injuste, faux, mensonge \\
\hline Dieu & Satan \\
\hline $\begin{array}{c}\left.\text { Vie [don de la vie, culture de vie } e^{43}\right] \\
\text { (Hétéroparentalité) }\end{array}$ & \begin{tabular}{c} 
Mort $\left[\begin{array}{c}\text { culture de mort, puisque les homosexuels } \\
\text { ne peuvent pas enfanter et que } \\
\text { la PMA n'est pas naturelle/avortement }] \\
\text { Homoparentalité }\end{array}\right.$ \\
\hline Mariage entre homme-femme
\end{tabular} \\
\hline & Déviances sexuelles \\
\hline Hétérosexualité & Mariage pour tous \\
\hline & Homosexualité \\
\hline & Homosexualisme, Homophilie, Homo-folie \\
\hline & Révolution sexuelle \\
\hline & Lobbies homosexuels \\
\hline & Pédophilie \\
\hline & Polygamie \\
\hline & Inceste \\
\hline
\end{tabular}

Tableau 3 : Les thématiques du corpus

42 C'est-à-dire une focalisation sur les termes, les opinions, les croyances et les arguments principaux.

43 "Culture de vie ", " culture de mort " sont des expressions employées par les opposants à l'interruption volontaire de grossesse, qualifiée d'acte de mort. Ces opposants défendent donc par extension, la vie, le droit de tout " enfant à naître " (expression pro-vie par laquelle les opposants à l'IVG " humanisent » le fotus rendant ainsi rendant l'acte d'avortement criminel, meurtrier). Il est à noter que l'apparition de ces termes propres au milieu " pro-vie " n'est pas anodine et s'explique par la très forte proximité des groupes associatifs contre le mariage pour tous et contre le droit à l'avortement. Les deux tendances associatives se rejoignent sur les réseaux traditionalistes. Le " mariage pour tous " entre donc, selon eux, dans une même logique posant un problème d'ordre civilisationnel. 
Ceci n'est qu'un extrait synthétique de l'opposition dualiste exprimée à travers le corpus. De la dichotomie faite entre le bien et le mal découle l'identification d'adversaires, qu'une des vidéos de notre corpus illustre : "Ils veulent nous imposer leur révolution sexuelle ${ }^{44}$. Cette vidéo énumère les principaux responsables d'une "déchéance " morale, qui sont : les lobbies homosexuels, les lobbies franc-maçons, les officines d'extrême gauche, les officines anti-racistes ${ }^{45}$, les réseaux mondialistes de la finance. D'autres responsables sont également récurrents dans le corpus : la laïcité, l'État et le gouvernement, les féministes ou encore les médias de gauche.

Ces adversaires sont illustrés par le champ sémantique de la manipulation : complotiste, machination, manipulation, coalition et identifiés par un "Eux " symbolique. L'ensemble des documents du corpus se structure sur une identification des responsables et une mise en avant des conséquences morales et civilisationnelles (ici du "mariage pour tous »). Ainsi la vidéo "Aujourd'hui le mariage homo, demain la polygamie " ${ }^{46}$ sur Gloria.tv, illustre le propos. Elle amène, par un procédé de répétition de l'expression "Le mariage : c'est un homme et une femme " et par son opposition cyclique à la notion de "polygamie ", à la conclusion que le non respect de la complémentarité homme-femme dans le mariage conduit à des comportements polygames et à une légalisation potentielle de l'inceste. Une « lecture flottante » des articles des blogs du corpus permet de se rendre compte que cette structure rhétorique se retrouve sur l'ensemble de la réinfosphère.

Les résumés de contenu élaborés durant l'analyse des textes et vidéos, montrent le lien entre le modèle familial prôné et Dieu et sont représentatifs de l'ensemble idéologique du réseau traditionaliste de mobilisation, ou de discours sur les raisons de la mobilisation. Les sites de la sphère cultuelle et de solidarité dont nous avons parlé en première partie insistent sur le lien entre le modèle familial et Dieu (suivre ce modèle familial rapproche du divin). Pour citer une femme dans une des vidéos de notre corpus ${ }^{47}$ : "C'est une offense incroyable aux lois de Dieu, souvenez vous Dieu, dans l'Ancien Testament a détruit Sodome et Gomorrhe, qui étaient deux villes homosexuelles. Si ces lois étaient votées on vivrait vraiment le choléra et la peste ». Les sites et

44 Vidéo de promotion d'un ouvrage d'Alain Escada (14/04/2013), http://www.civitas-institut. com/content/view/954/1/ réalisée par Jacques Buffet sur YouTube, [consultée le 16/04/2013 et publiée la veille].

45 «Antiraciste » désigne un manque de considération en ce qui concerne le "racisme anti-blanc». Certains sites comme "L'observatoire de la Christianophobie » ou l'AGRIF «Alliance générale contre le racisme et pour le respect de l'identité française et chrétienne " en font un usage régulier. Le terme est à mettre en relation avec ceux de " cathophobie » et "christianophobie ".

46 Disponible sur Gloria tv entre autres http://fr.gloria.tv/?media=334978 [consultéle 20/10/2013].

47 "Prière de rue devant le Sénat. L'homosexualité, le choléra et la peste ", Civitas et Christ-roi, YouTube, publiée le 5/04/2013. 
blogs traditionalistes politiquement positionnés ciblent leur argumentation davantage sur les aspects sociétaux et civilisationnels et les blogs militants insistent sur l'importance de l'action. Les références symboliques employées dans ces discours se trouvent également représentées par l'emploi de l'image. Les symboles visuels délimitent un espace d'appartenance, un territoire symbolique et identifient un groupe.

Ainsi, nous faisons état de trois principales utilisations de l'image : l'emploi de symboles propres au catholicisme et en particulier au traditionalisme. Généralement placés en page d'accueil ces symboles peuvent être le glaive, le sacré cœur de Jésus Christ-Roi, la croix vendéenne ou encore la fleur de lys. Ils sont des symboles catholiques et royalistes. Il y a également une récupération de symboles de résistance (en dehors du champ catholique) : tels que les personnages historiques Jean Moulin ou Gandhi ou encore le symbole du point levé. Enfin, nous trouvons une importante utilisation d'images victimaires destinées à ériger une martyrologie $e^{48}$ : cela concerne principalement des images révélant une "brutalité policière " lors de manifestations anti-mariage pour tous.

Nous assimilons cette récupération à un effet miroir $^{49}$ qui favorise une prise de conscience et une " détermination collective $"{ }^{50}$ et mobilise des références communes au traditionalisme. L'objectif des images employées est de servir la cause en la légitimant, et d'accompagner un discours destiné semble-t-il à rompre le lien social entre les individus qui suivent l'ordre naturel du concept familial et le reste de la société considérée comme corrompue. Elles ont également pour but de rassembler derrière des références symboliques plus larges que le champ restreint du traditionalisme catholique et s'engagent dans des procédés de comparaison à des mobilisations activistes historiques ou actuelles. Le groupe des hommen imitant les codes protestataires des activistes femmen illustre ce phénomène : tout en étant antiféministes les hommen emploient les codes des femmen comme la semi-nudité et les inscriptions revendicatrices sur le torse.

Nous constatons ensuite que le réseau traditionaliste partage une représentation du modèle familial en accord avec un imaginaire préconciliaire de la Tradition catholique, terreau de la mobilisation. Le bloggeur traditionaliste s'exprime rarement en son nom, il parle d'un "nous " symbolique mettant en avant la figure héroïque du martyr catholique, exaltant ainsi la fibre guerrière $\mathrm{du}$ lecteur. Tout un champ lexical de la guerre (combat, rang, adversaire) se développe autour du sentiment de perte de repères, de sens et d'appartenance à

48 La pratique est extensible à la cause pro-vie. Les associations pro-vie emploient de manière courante des images de fœetus morts ou de nourrissons mort-nés, destinées à susciter l'indignation et dénonçant le génocide perpétré.

49 Antonio Casilli, Journée d'études « Sacrés liens! ", Étudier les liens en sciences sociales des religions, EHESS, Paris, 21 mai 2013.

50 Terme employé dans l'intitulé d'un post du blog Le Salon Beige en date du 29 mars 2013 en parlant de la dernière manifestation ayant eu lieu. 
ce qui n' est plus. La blogosphère d'information joue le rôle de pilier central dans le réseau traditionaliste, entre l'action symbolique par le discours sur Internet et les actions concrètes. Ce phénomène peut s'expliquer par la mobilisation de ressources historiques du traditionalisme, telles que les théories du complot judéo-maçonnique, autant que par la production de réflexions actuelles sur les études de genre (ou " théorie du genre " pour les catholiques traditionalistes) $)^{51}$.

La structure rhétorique et les éléments du discours mis en avant témoignent d'une homogénéité dans le système de représentations de la " réinfosphère ". L'imbrication des blogs qui s'observe à travers le référencement de blogrolls principalement, confirme l'appartenance à un ensemble référentiel commun et suggère une intercompréhension des enjeux civilisationnels mis en avant. La « réinfosphère » semble faire apparaître des « règles du jeu » communicationnelles propres au milieu concerné, dans une veine traditionnelle, polémiste et parfois pamphlétaire. Nous pourrons peut-être formuler l'hypothèse de l'existence sur cette blogosphère d'une "situation idéale de parole " ${ }^{52}$ remplissant les conditions des possibilités de l'intercompréhension traditionaliste. Une seconde hypothèse sera formulée à l'issue du traitement du contenu. C'est celle de l'émergence au sein du milieu traditionaliste, du moins dans son expressivité numérique, d'identités que Manuel Castells qualifierait de "défensives ", c'est-à-dire construites dans un mouvement de réaction à une logique dominante et d'effondrement d'un cadre familial hétéro-normé centré sur des valeurs traditionnelles (Dieu, Nation, Famille) ${ }^{53}$.

\section{Communication organisationnelle, vers une mobilisation effective}

La mobilisation traditionaliste est d'abord soutenue par un militantisme discursif partagé en réseau. L'action se mène par la diffusion de l'information à partir de la blogosphère de réinformation. Cette mobilisation symbolique (faisant appel à un imaginaire identifié) participe au renforcement d'une identité minoritaire existante en même temps qu'elle élargit sa réception en empruntant des références communes à l'ensemble de la société et fait appel à une mémoire collective. Parmi les acteurs du web traditionaliste, quel est le rôle des institutions traditionalistes? Au regard des réseaux mis en avant nous pouvons nous poser la question suivante :

Mais alors où sont les organisations religieuses ? Dans la mesure où ces dénominations, églises, communautés, sectes ou cultes manifestent une inscription sociale et une permanence dans l'actualisation de croyances religieuses, de pratiques

51 Anthony Favier, précisions sur le blog " Penser le genre catholique », http://penser-le-genrecatholique.over-blog.com/ [consulté le 23/10/2013].

52 Jürgen Habermas, Théorie de l'agir communicationnel, t. 2, Pour une critique de la raison fonctionnaliste, Paris, Fayard, 2014 (1987).

53 Manuel Castells, L'ère de l'information, t. 2, Le pouvoir de l'identité, Paris, Fayard, 1999. 
rituelles et morales, le tout faisant appel à une symbolique qui les fonde comme groupes de croyants, de fidèles, de disciples, d'adeptes, on peut dire que le groupe religieux évolue selon un développement organisationnel de type "normatif ». [...] Les critères de ce développement retenus ici sont une fonction de leadership qui oriente une cohésion minimale des croyances et des pratiques, assure une continuité dans le temps pour assurer son remplacement, instaure un contrôle des statuts et des rôles établissant les relations entre les membres et la sociétéét.

Paul Stryckman s'exprime ainsi avec l'objectif de dresser des éléments pour l'" analyse organisationnelle des pratiques religieuses ". Or, sommes-nous face ici à des pratiques religieuses à proprement parler ? Bien que concernant des institutions religieuses et des ensembles de fidèles, le sujet est-il concrètement tourné vers la communication organisationnelle de groupes religieux en tant que telle ? Après avoir mené une analyse morphologique et typologique des réseaux il semble que les acteurs impliqués dans un discours religieux normatif soient des laïcs et des acteurs associatifs. De même l'essentiel du discours incitant à une mobilisation est produit par ces derniers. Une recherche précédente a établi que les institutions religieuses à travers leurs sites internet fournissent l'essentiel de l'information et de l'enseignement théologique et liturgique ${ }^{55}$. En revanche ces mêmes laïcs sont, nous l'avons vu, impliqués dans la production et la diffusion d'un savoir (standardisé) à l'attention de communautés de croyants. Nous pouvons l'affirmer notamment de par la structure même du réseau traditionaliste. Ainsi ils sont producteurs d'un discours normé, en vue d'une mobilisation (collective) contre le " mariage pour tous ". L'action collective se structure sur la capacité d'un groupe à s'organiser et à communiquer. Les acteurs concernés savent qu'il est nécessaire d'être actif sur Internet pour élargir les lieux traditionalistes (même symboliques), augmenter l'audience, élargir le champ de réception, puis fidéliser les internautes. Les traditionalistes s'exercent à la communication par le biais de formations disponibles sur les sites d'associations (" Je me prépare à débattre » sur le site de la « manif pour tous » a son équivalent sur les autres sites militants). L'internaute militant peut s'il le souhaite se former à des techniques d'argumentation en faveur de la lutte contre le «mariage pour tous ».

La réinfosphère semble remplir les critères de développement organisationnel énoncés par Stryckman, à savoir la cohésion, la continuité et le contrôle. La cohésion correspond ici à la diffusion normative et homogène d'une idéologie héritée et réactivée en contexte de "mariage pour tous " $"$;

54 Paul Stryckman, op. cit., p. 2-3.

55 Charlotte Blanc, "Traditionalisme catholique et éducation-médias. Catéchèse et évangélisation ", Actes du colloque international sur l'Éducation-médias, Nouveaux enjeux, rôles et statuts des acteurs, Abidjan, 13-14 mars 2014, UCAO, à paraître chez L'Harmattan.

56 Notons tout de même que cette « réactivation " que nous évoquons a été une réalité au moment du PACS et de la loi sur l'IVG. Elle n'est donc pas nouvelle. Toutefois les réactions à la loi sur le « mariage pour tous » ont suscité une implication communicationnelle et une action collective plus importantes que précédemment. 
l'exercice de formation des internautes à l'argumentation anti-mariage pour tous confere une certaine continuité à la démarche ; tandis que le contrôle au sein des communautés s'assure partiellement par un contrôle à l'entrée sur certains site. Il y a en effet une logique d'inclusion-exclusion qui s'installe sur certaines plateformes, c'est le cas du réseau MISSA pour lequel l'internaute ne peut s'inscrire que s'il a des connaissances parmi les membres de l'institution religieuse. La mobilisation informationnelle s'effectue à travers deux tendances, une communication externe visant à améliorer l'image de la cause défendue et une interne, devant renforcer une communauté d'appartenance. La communication des groupes traditionalistes est diversifiée. Elle permet aux internautes de communiquer sur le Net, donc de participer au débat via les forums confessionnels, par les blogs et les pages des associations sur les réseaux sociaux. La communication traditionaliste expose les enjeux de la cause et offre une information alternative. Ainsi, la transmission d'une idéologie à travers la sphère de réinformation est un acte d'organisation autour de valeurs et de projets communs. Malgré les divergences d'opinions au sein de la mouvance traditionaliste, les associations exploitent un même réservoir symbolique et des pratiques similaires de communication à travers les formations en ligne ou organisées en ligne et les rencontres entre associations et institutions.

Cette contribution propose d'aborder les milieux traditionalistes sur le web. Elle s'applique à questionner les possibilités méthodologiques de recherche sur des groupes religieux radicaux en ligne et interroge dans un sens plus large l'élaboration d'un corpus sur le terrain poreux qu'est le web. Nous pouvons dresser quelques premiers constats, ou du moins, fournir des pistes de réflexion. L'enjeu du sujet est multidimensionnel et recoupe tant la question des nouveaux espaces délibératifs et de participation en ligne, que la place du religieux dans la sphère publique ainsi que la communication religieuse en soi. Nous sommes donc face à un objet complexe. Nous avons souhaité focaliser notre attention sur les éléments structurant des réseaux traditionalistes en centrant notre recherche sur la blogosphère dite de réinformation. Cette dernière, nous pouvons l'avancer, constitue un véritable organe idéologique de réflexion politico-religieuse produite par des acteurs intentionnellement tournés vers une vision alternative des médias, justifiant un usage stratégique des liens hypertextes comme ressource symbolique.

L'analyse de ces liens hypertextes et des blogrolls a permis de faire émerger une importante blogosphère qui, après une analyse de contenu, s'est révélée fournir une (ré)information relativement homogène, quels que soient les vidéos et textes sélectionnés sur les divers blogs du corpus. Ainsi, cette homogénéité discursive, l'intentionnalité communicationnelle des acteurs sur des sujets de société (" mariage pour tous " dans le cas présent), l'uniformité des cadres d'énonciation, ainsi que la centralité de la blogosphère de réinformation 
dans les réseaux traditionalistes suggèrent une co-construction de sens dans l'interaction. La structure communicationnelle des réseaux traditionalistes, qui mériterait une recherche plus approfondie, témoigne d'une volonté de mise en commun et de structuration de l'action collective qui ne passe pas directement par des acteurs institutionnels. En effet, nous sommes déjà ici dans ce qui relève de l'action coordonnée, collective dans la mesure où le récit d'une action se révèle être tout aussi agissant que l'action elle-même. Ce postulat nous permettra ultérieurement de produire une analyse des discours sur l'action effective des traditionalistes (à travers les récits de manifestations par exemple).

Ici les traditionalistes élaborent un ensemble de récits autour d'un même fait lu à la lumière d'une perception spécifique de ce à quoi renvoie la Tradition. La récurrence de la dialectique Bien-Mal structure le récit et est révélatrice d'un imaginaire traditionaliste homogène, conforme à l'état de l'art existant sur la question. Ainsi, la blogosphère co-construit un modèle représentationnel qui répond semble-t-il aux attentes des lecteurs. Enfin, la recherche engagée permettra dans l'avenir d'envisager le terrain traditionaliste par le biais d'une approche complexe de l'hypertexte sur l'architecture du web catholique.

Charlotte Blanc EA 4426 MICA

Université Bordeaux Montaigne charlotteblanc@outlook.fr

\begin{abstract}
Résumé
L'article a pour objectif la mise en évidence de la structure des réseaux de communication des traditionalistes catholiques sur Internet. La recherche s'est construite sur la base des liens hypertextes des sites et par le truchement du thème du " mariage pour tous ». Ce dernier, employé comme moyen d'accès au réseau traditionaliste a permis de mettre au jour certaines caractéristiques identitaires et idéologiques de cette mouvance religieuse et de questionner l'homogénéité du réseau. Ainsi, l'article expose la morphologie du web traditionaliste et dresse un début de typologie des blogs de la blogosphère.
\end{abstract}

\title{
Mots-clés
}

Traditionalisme catholique, Internet, réseaux, blogosphère, « mariage pour tous ».

\begin{abstract}
This paper deals with the presence of catholic traditionalists on the Internet and tries to enlighten the communication networks this religious movement have built on the web. This research, by using hyperlink indicators and homosexual marriage topic, intends to identify the morphological and typological caracteristics of the traditionalist blogosphere.
\end{abstract}

\section{Keywords}

Catholic traditionalism, Internet, networks, blogosphere. 